\title{
Diagnostic accuracy of optical coherence tomography angiography for choroidal neovascularization: a systematic review and meta-analysis
}

\author{
Rui Wang ${ }^{1}$, Zhenzhen Liang ${ }^{2}$ and Xin Liu ${ }^{1 *}$ (I)
}

\begin{abstract}
Background: Optical coherence tomography angiography (OCTA), an innovative image technique, renders visualization of ocular neovascularization through non-invasive means, which has been applied in recent years. Therefore, the present study was designed to assess the diagnostic value of OCTA in detecting the choroidal neovascularization (CNV).

Methods: In brief, PubMed, Web of Science and Cochrane Library databases were systematically searched from January 2014 to June 2019. Afterwards, a meta-analysis was performed to determine the pooled diagnostic accuracy in a random-effects model using STATA 15.1 and Meta-Disc 1.4 software. Quality Assessment of diagnostic Accuracy Version 2 was used to evaluate the risk of bias of each study by Revman 5.3 software. In addition, a meta-regression model was further conducted to explore potential sources of heterogeneity.

Results: According to pre-set inclusion and exclusion criteria, 16 eligible studies were enrolled in this study. A total of 447 CNV eyes and 414 non-CNV eyes were included to investigate the diagnostic accuracy of OCTA. As a result, the pooled sensitivity, specificity, positive likelihood ratio (PLR), negative likelihood ratio (PLR), diagnostic odds ratio (DOR) and the area under the summary receiver operating characteristic curve (sROC-AUC) were 0.87 (95\% Cl 0.81-0.92), 0.97 (95\% Cl 0.92-0.99), 32.7 (95\% Cl 10.1-105.5), 0.13 (95\% Cl 0.08-0.20), 252 (95\% Cl 63-1011) and 0.96 (95\% Cl 0.94-0.97), respectively.
\end{abstract}

Conclusions: In summary, we demonstrated that OCTA was of high diagnostic value for detecting intraocular CNV.

Keywords: Optical coherence tomography angiography, Choroidal neovascularization, Diagnosis, Meta-analysis

\section{Background}

Optical coherence tomography angiography (OCTA), based on optical coherence tomography (OCT), is an advanced imaging technology which has recently been approved for clinical ophthalmology application [1]. OCTA is considered as a noninvasive, rapid, secure and repeatable method, which is capable of displaying retinal vasculatures in threedimensional assessment [2]. Without applying intraocular contrast agents, OCTA, a motion contrast imaging modality, depends on two sorts of backscattered light signals to visualize ophthalmic vascular networks in high-resolution.

\footnotetext{
*Correspondence: liuxinjlu01@163.com

${ }^{1}$ Epidemiology and Statistics, School of Public Health, Jilin University,

Changchun 130021, China

Full list of author information is available at the end of the article
}

To be specific, one of the two signals is stable and derives from immobile structures such as neurosensory tissue, while the other is variable and dynamic over time generated by constantly moving tissues like erythrocytes. In comparison with stationary areas, erythrocytes can be measured by repeated B-scans. Thus, an image is created by detecting continuous changes of blood flow. This dye-less imaging technology renders confirmed diagnosis of ocular diseases without the restriction generated by contrast agents.

Pathological myopia, central serous chorioretinopathy (CSCR), age-related macular degeneration (AMD) and uveitides are commonly-detected diseases in ophthalmology, which are characterized by the formation of choroidal neovascularization (CNV) [3-6]. In these disorders, neovascularization is commonly formed in the 
retina under specific stimulation $[7,8]$. Neovascularization is highly permeable, in which hemorrhagic or exudation will occur with the progression of diseases. Under these conditions, visual impairment and irreversible blindness might present in the case of further exacerbated pathological changes.

The prevalence of ophthalmological diseases including pathological myopia and AMD is increasing year by year, which might lead to a potential risk of blindness in the population [9-11]. Severe deterioration of visual acuity will occur by degrees if the progression of CNV is not prevented [12]. To reduce adverse consequences, early detection of CNV will be conducive to increase opportunities for timely and proper treatment. In addition, noninvasive imaging technology is also used for longterm monitoring on eyes which are at high risk of developing CNV [13]. In clinical practice, there are many diagnostic tools for ocular diseases including funduscopy, fluorescein angiography (FA), indocyanine green angiography (ICGA), conventional OCT and OCTA. As a new imaging method, OCTA shows advantages in detecting CNV [14]. In spite of the reported diagnostic accuracy of OCTA for CNV, results are inconsistent with no solid data confirming the exact accuracy of this method. To this end, this study was designed to evaluate the overall diagnostic value of OCTA in the detection of CNV by analyzing different studies.

\section{Methods}

\section{Search strategy and selection criteria}

This study was conducted in accordance with Preferred Reporting Items for Systematic Reviews and Meta-analysis
(PRISMA) guidelines [15]. The clinical application of OCTA was first reported in 2014, thus, the initial year of publication was confined to 2014. Three electronic databases including PubMed, Web of Science and Cochrane Library were systematically retrieved by using the following key terms: 'Optical Coherence Tomography Angiography' or 'Optical Coherence Tomography Angiogram' or 'OCTA' and 'Choroidal Neovascularization' or 'Choroid Neovascularization' or 'CNV'. Two reviewers screened literatures independently and the deadline of this searching was set at June 2019.

The inclusion criteria were as follows: (1) eyes diagnosed as CNV were included regardless of etiologies; (2) all types of CNV were supposed to be recruited; (3) OCTA was the diagnostic method for $\mathrm{CNV}$ compared with at least one dye angiography; (4) sufficient data were available to calculate the true positive, false positive, false negative and true negative.

The exclusion criteria were as follows: (1) conference abstracts, editorials, case reports, letters, reviews and experimental studies; (2) full-text of published articles was not written by English.

\section{Data extraction}

Initial data from each eligible literature were extracted independently by two investigators according to pre-determined research needs: year of publication, first author, country, ethnicity, type of study, number of eyes, etiology of CNV, reference standard, type of CNV, type of OCTA device and diagnostic performance (a true positive, false positive, false negative and true negative).

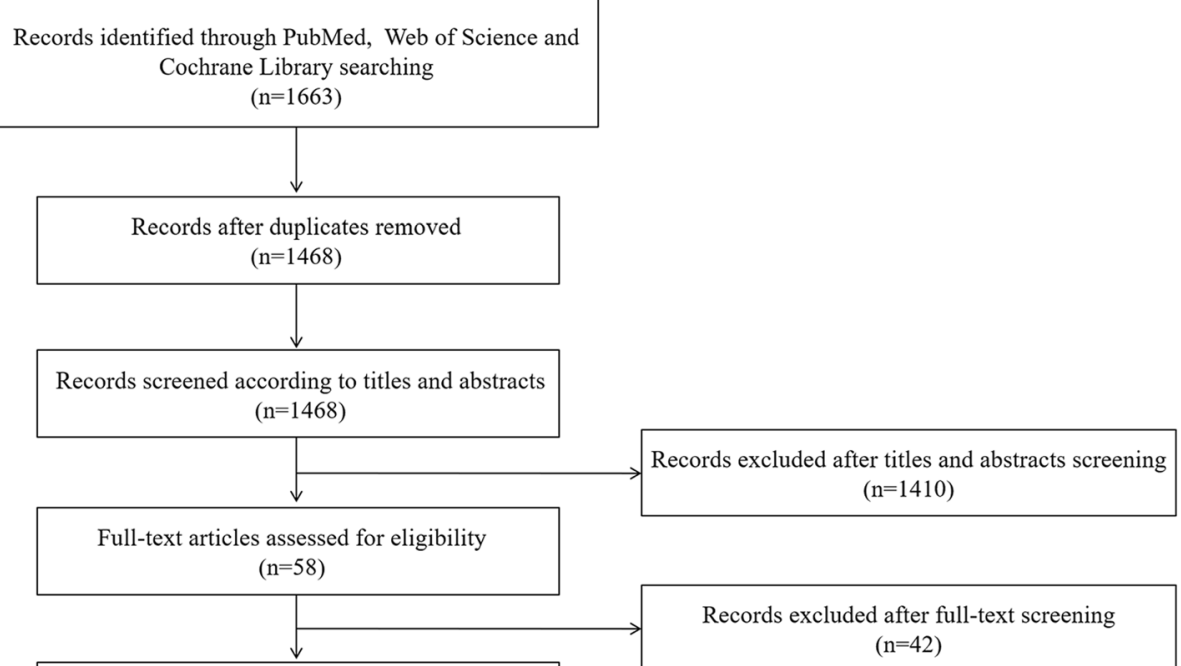

Studies included in qualitative synthesis $(n=16)$

Fig. 1 Flow diagram of the selection of studies 
In the case of discrepancy, a third reviewer was consulted to achieve consistent conclusions.

\section{Quality assessment}

Quality Assessment of diagnostic Accuracy Version 2 (QUADAS-2) was used to assess quality of each eligible study [16]. Four domains in QUADAS-2 tool included patient selection, index test, reference standard, and flow and timing. Each item of these domains was required to quantitatively evaluate one by one, which was attentively conducted by two independent investigators through full-text reading. Different results were resolved through consensus by a third reviewer.

\section{Statistical methods}

Above all, the evaluation of threshold effect was conducted according to the Spearman correlation coefficient. A $P$-value less than 0.05 possibly indicated a significant threshold effect. A random-effects model was more suitable to analyze small sample studies. Publication bias was investigated by Deeks' funnel plot qualitatively and assessed by $P$-value quantificationally. Additionally, a $P$-value over 0.05 suggested no significant publication bias. To be specific, the pooled sensitivity, specificity, positive likelihood ratio (PLR), negative likelihood ratio (NLR) and diagnostic odds ratio (DOR) were major diagnostic evaluation indicators. The summary receiver operator characteristic (sROC) curve was accurately drawn so as to calculate the area under the sROC curve (sROCAUC). Moreover, Fagan's Nomogram was conducted to analyze the clinical valuation. For a positive diagnosis, the higher post-test probability than the pretest probability indicated the great value of the specific method for a definite diagnosis. Otherwise, for a negative diagnosis, a lower post-test probability

Table 1 Major characteristics of included studies

\begin{tabular}{|c|c|c|c|c|c|c|c|c|c|c|c|c|}
\hline Author & Year & $\mathrm{Tp}$ & $\mathrm{Fp}$ & $\mathrm{Fn}$ & $\mathrm{Tn}$ & Country & Ethnicity & Type of study & Etiology & Reference image & Type of CNV & Device \\
\hline Moult E & 2014 & 16 & 0 & 1 & 63 & USA & Caucasian & Prospective & AMD & FA,ICGA & NA & VCSEL \\
\hline Lupidi M & 2015 & 4 & 0 & 1 & 20 & France & Caucasian & Retrospective & AOFVD & FA,ICGA,OCT & NA & Spectralis \\
\hline $\begin{array}{l}\text { Bonini Filho } \\
\text { MA }\end{array}$ & 2015 & 8 & 0 & 0 & 19 & USA & Mixed & Prospective & CSCR & FA & Type1, mixed type $1 / 2$ & AngioVue \\
\hline de Carlo TE & 2015 & 4 & 2 & 4 & 20 & USA & Mixed & Retrospective & Multiple1 & FA & NA & AngioVue \\
\hline Shaimov TB & 2015 & 33 & 1 & 4 & 14 & Russia & Caucasian & Prospective & AMD & FA & Type1,2 & AngioVue \\
\hline Carnevali A & 2016 & 18 & 0 & 4 & 22 & Italy & Caucasian & NA & AMD & ICGA & NA & $\begin{array}{l}\text { AngioPlex/ } \\
\text { Angiovue }\end{array}$ \\
\hline Miyata M & 2016 & 16 & 0 & 1 & 4 & Japan & Asian & NA & Myopia & FA & Type 2 & AngioVue \\
\hline de Carlo TE & 2016 & 6 & 1 & 1 & 22 & USA & Caucasian & Retrospective & CSCR & FA & NA & AngioVue \\
\hline Gong J & 2016 & 45 & 11 & 7 & 23 & China & Asian & Retrospective & AMD & FA & Type1,2,mixed type1/2 & AngioVue \\
\hline Querques L & 2017 & 19 & 2 & 2 & 30 & Italy & Caucasian & Retrospective & Myopia & FA,OCT & NA & AngioPlex \\
\hline Faridi A & 2017 & 32 & 0 & 0 & 40 & USA & Caucasian & Prospective & AMD & FA,OCT & NA & AngioVue \\
\hline Ahmed D & 2018 & 81 & 0 & 26 & 49 & Austria & Caucasian & Retrospective & AMD & FA & $\begin{array}{l}\text { Type1,2,3, } \\
\text { mixed } \\
\text { type }\end{array}$ & Topcon \\
\hline $\begin{array}{l}\text { Nikolopoulou } \\
\text { E }\end{array}$ & 2018 & 44 & 2 & 6 & 18 & Italy & Caucasian & Prospective & AMD & $\mathrm{FA}$ & $\begin{array}{l}\text { Type1,2,3,mixed } \\
\text { type1/2 }\end{array}$ & AngioVue \\
\hline Souedan V & 2018 & 8 & 1 & 1 & 6 & France & Caucasian & Retrospective & Multiple2 & FA,OCT & All types & AngioVue \\
\hline Soomro T & 2018 & 32 & 6 & 13 & 26 & England & Caucasian & Retrospective & Multiple3 & FA & Type1,2 & Spectralis \\
\hline de Oliveira T & 2019 & 9 & 0 & 1 & 12 & Brazil & Mixed & NA & Multiple4 & $\mathrm{FA}, \mathrm{ICGA}, \mathrm{OCT}$ & NA & AngioVue \\
\hline
\end{tabular}

Tn True positive, Fp False positive, Fn False negative, Tn True negative, AMD Age-related macular degeneration, AOFVD Adult onset foveomacular vitelliform dystrophy, CSCR Central serous chorioretinopathy, FA Fluorescein angiography, ICGA Indocyanine green angiography, OCT Optical coherence tomography, CNV Choroidal neovascularization

Multiple1 = AMD, CSCR and different diagnosis (angioid streaks, multifocal choroiditis, myopic degeneration, pars planitis, or an unclear diagnosis with the

differential diagnosis including CSCR, neo-vascular AMD, or polypoidal choroidal vasculopathy (PCV)

Multiple2 $=$ AMD, reperfused central retinal artery obstruction, one adult onset pseudovitelliform lesion, drusenoid pigment epithelium detachment, CSCR,

macroaneurysm and reticular dystrophy

Multiple3 $=$ AMD, PCV, CSCR or pathological myopia

Multiple4 = AMD, PCV and CSCR

VCSEL = OCTA combining vertical cavity surface emitting laser (VCSEL) swept light source OCT with $400 \mathrm{kHz}$ A-scan rate prototype system

Topcon = Topcon OCT-A (Topcon Corporation, Tokyo, Japan)

AngioVue = AngioVue OCT-A (Optovue, Inc., Freemont, CA)

AngioPlex = AngioPlex OCT-A (Carl Zeiss Meditec, Inc., Dublin, USA)

Spectralis = Spectralis OCT-A (Heidelberg Engineering, Heidelberg, Germany)

$\mathrm{NA}=$ Not available 


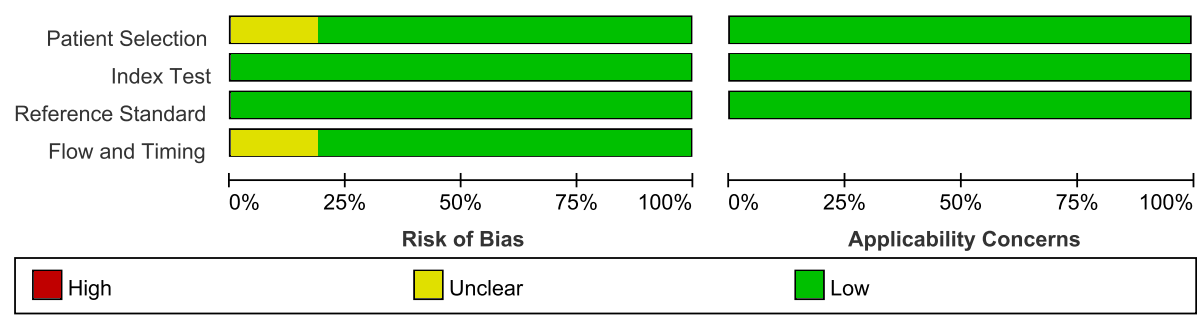

Fig. 2 Methodological quality graph by QUADAS-2 of 16 articles

contributed to the identification of healthy individuals compared with the pre-test probability. In order to obtain reliable results, statistical heterogeneity was supposed to be analyzed by evaluating $I^{2}$ and $P$-value quantificationally. If the value of $I^{2}$ was more than $50 \%$ or $P$-value was less than 0.05 , there was significant heterogeneity among these studies. Therefore, a meta-regression model was used to analyze sources of heterogeneity by adding covariates. Heterogeneity analysis of sensitivity and specificity was presented separately. Moreover, a joint model was performed to find out sources of overall heterogeneity. A $P$-value less than 0.05 indicated that this covariate might be the source of heterogeneity. Stata 15.1 and Meta-Disc 1.4 were used for statistical analyses in this meta-analysis. Besides, Revman 5.3 software was implemented to assess the quality of each included literature by QUADAS-2 tool.

\section{Result}

Study selection and study characteristics

As a result, a total of 1663 papers were identified (PubMed: 139, Web of Science: 1473 and Cochrane Library: 51) by using pre-defined search strategy (Fig. 1). According to inclusion and exclusion criteria, 58 fulltext articles were finally screened. In total, 16 eligible studies including $447 \mathrm{CNV}$ eyes and 414 non-CNV eyes with sufficient data were selected into the final analysis [17-32]. There were five prospective studies and eight retrospective studies, while the remaining three studies failed to show the type of study. The ethnicity was Asian in two articles while that of other 14 articles was

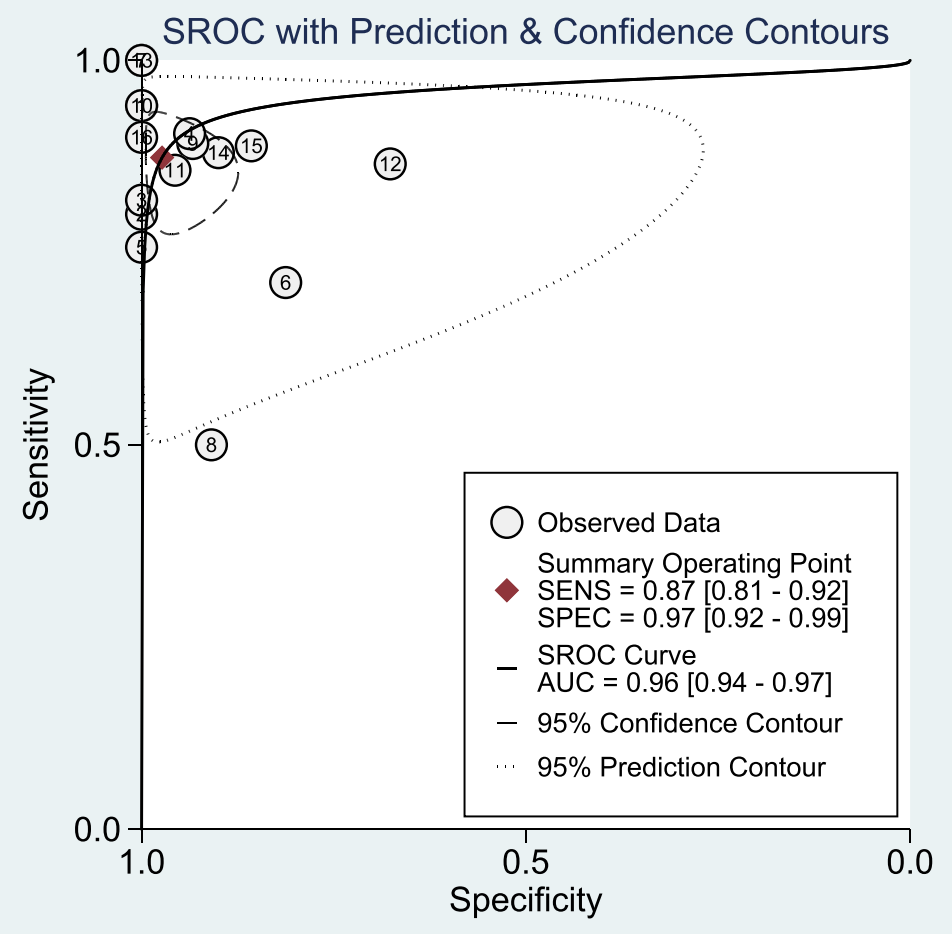

Fig. 3 Diagram of sROC curve for assessing the diagnostic value of OCTA in detecting CNV 
Caucasian or mixed. Sample size of cases ranged from 5 to 107 in each study, which varied from 4 to 63 for controls. In total, the size of eyes sample in eight studies was less than 50, while it reached to more than 50 in the other eight studies. In addition, OCTA was only compared with FA in nine studies, while OCTA was compared with other diagnostic techniques such as ICGA or multimodal imaging in the other seven studies. Device types included AngioVue OCTA (10 studies), Spectralis OCTA (2 studies), AngioPlex OCTA (1 study), Topcon OCTA (1 study). In addition, an earlier study in 2014 used OCTA combining vertical cavity surface emitting laser swept light source OCT with $400 \mathrm{kHz}$ A-scan rate prototype system. Besides, in another study, two types of OCTA devices were utilized, including AngioPlex OCTA and AngioVue OCTA. The etiologies of these $\mathrm{CNV}$ were various including $\mathrm{AMD}$, CSCR, adult onset foveomacular vitelliform dystrophy, pathological myopia, reperfused central retinal artery obstruction, adult onset pseudovitelliform lesion, drusenoid pigment epithelium detachment, macroaneurysm, reticular dystrophy, polypoidal choroidal vasculopathy and so on. Of note, all kinds of CNV were included in this study (Table 1).

\section{Quality assessment and publication bias}

The quality assessment of each study was performed by using QUADAS-2 (shown in Fig. 2), indicating that quality of these studies were almost at moderate to high levels. In order to evaluate the publication bias, Deeks' funnel plot asymmetry test was conducted. Consequently, a $P$-value of 0.71 suggested no significant publication bias among included studies.

\section{Diagnostic performance and clinical value}

The pooled diagnostic performance of OCTA in detecting CNV showed 0.87 (95\% CI $0.81-0.92$ ) of sensitivity, 0.97 (95\% CI $0.92-0.99)$ of specificity and 252 (95\% CI 63-1011) of DOR. The sROC curve was showed in Fig. 3, which was 0.96 (95\% CI 0.94-0.97). Besides, PLR and NLR were 32.7 (95\% CI 10.1-105.5) and 0.13 (95\% CI $0.08-0.20)$, respectively. According to PLR and NLR, the Fagan's Nomogram was used to obtain $88 \%$ of positive post-test probability and $3 \%$ of negative post-test probability, when 0.20 of pre-test probability was established in advanced (Fig. 4).

\section{Heterogeneity analyses}

Moreover, the Spearman correlation coefficient was 0.166 and $P$-value was 0.540 , thus, no significant threshold effect existed in this analysis. The $I^{2}$ value of $86 \%$ with $P$-value $<0.05$ suggested significant overall heterogeneity. The forest plots of sensitivity and specificity was conducted, revealing that the $I^{2}$ of

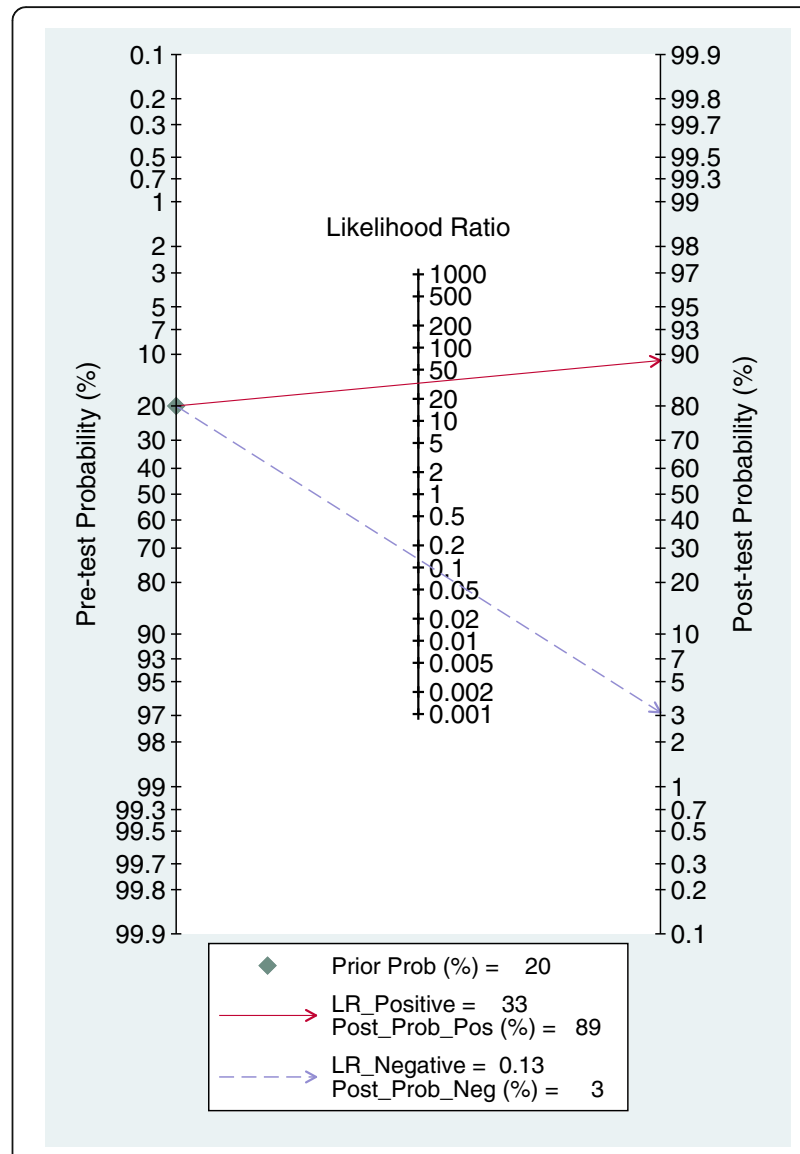

Fig. 4 Fagan's Nomogram of OCTA for CNV

sensitivity and specificity were 71.37 and $82.20 \%$, respectively (Fig. 5). Therefore, a meta-regression was conducted to perform sources of heterogeneity for analysis of the non-threshold effect. As a result, ethnicity (only Asian / other races), type of study (prospective / retrospective), etiology of CNV (only AMD / other ocular diseases), device of OCTA (only AngioVue / other devices), sample size $(\geq 50 /<50)$ were not potential sources of heterogeneity in sensitivity $(P$-values $\geq 0.05)$. However, $P$-value of reference standard (only FA / other reference standards) was less than 0.01 indicating that it might be a significant source of heterogeneity in sensitivity. The sensitivity of only FA standard group was lower than that of other standards group $(0.83$ versus $0.92, p<0.01)$. In terms of specificity, sources of heterogeneity were not found in this study. The results were shown in Table 2 .

To explore sources of overall heterogeneity, the joint model of meta-regression was established. Consequently, types of both study and device were potential sources of overall heterogeneity ( $p<0.01$ and $p=0.04$, respectively), while ethnicity, etiology, reference standard and sample size were not significant sources of overall heterogeneity ( $p=0.10, p=0.80, p=0.06$ and $p=0.49$, respectively). 


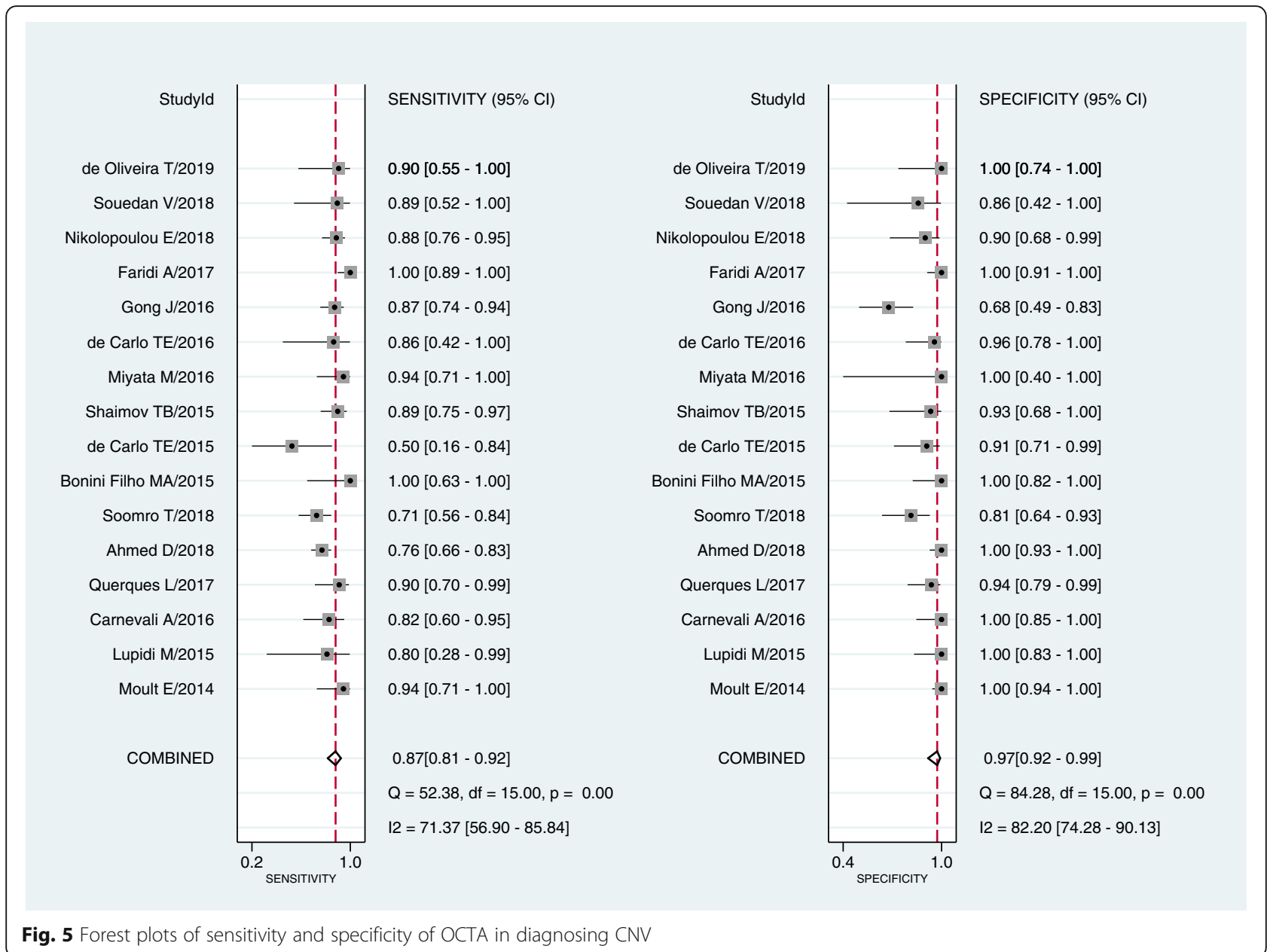

\section{Discussion}

Due to the advantages of OCTA in detecting microvascular pathology at various depths, previous studies had focused on the exploration of abnormal vascularity in the eyes by using OCTA images, however, these diagnostic performances failed to reach a consistent conclusion. In 2014, Moult $\mathrm{E}$ et al first reported the diagnostic value of OCTA in detecting CNV [17], revealing that 16 of $17 \mathrm{CNV}$ eyes in exudative AMD patients were clearly visualized on OCTA images in comparison with 63 eyes from healthy individuals. The sensitivity and specificity of this exploration were 94 and $100 \%$, respectively. Afterwards, De Carlo TE et al reported only four out of eight CNV eyes were clearly detected on OCTA, while all of the eight CNV eyes were definitely diagnosed in the article of Bonini Filho MA et al [19, 20]. Later, in 2016, Carnevali A et al in Italy and Gong $\mathrm{J}$ et al in China reported the diagnosis of CNV based on OCTA imaging [22, 25]. The sensitivity and specificity in the study of Carnevali A et al were 81.8 and $100 \%$, respectively, while they were 86.5 and $67.6 \%$, respectively, in the study of Gong J et al. Subsequently, Ahmed D et al, Faridi A et al and Nikolopoulou $\mathrm{E}$ et al also investigated the value of OCTA in detecting CNV in AMD [27-29]. As a result, the sensitivity of these three studies ranged from 75.7 to $88 \%$ and the specificity varied from 90 to $100 \%$. Herein, our meta-analysis analyzed the pooled efficacy of OCTA in detecting $\mathrm{CNV}$, showing that the sensitivity, specificity and sROC-AUC were $0.87,0.97$ and 0.96 , respectively. Therefore, OCTA technology might render rapid and safe exclusion of non-CNV eyes with high specificity for physicians.

In the elderly, AMD seems to be a major cause for visual impairment and the prevalence of AMD increases with age [33]. Due to the undefined cause of AMD, vascular endothelial growth factor (VEGF) inhibitors are widely applied in most treatments of AMD to prevent the growth of CNV [34]. Therefore, a useful method for monitoring the occurrence of $\mathrm{CNV}$ in $\mathrm{AMD}$ patients contributes to postponing the deterioration of this disease [35]. Currently, dye angiography is still used as a diagnostic golden standard for CNV; however, it is an 
Table 2 Meta-regression and subgroup analyses for sensitivity and specificity

\begin{tabular}{|c|c|c|c|c|c|c|}
\hline Covariate & Number of studies & Number of eyes & Sensitivity (95\% Cl) & $P$-value & Specificity (95\% Cl) & $P$-value \\
\hline \multicolumn{7}{|l|}{ Ethnicity } \\
\hline Asian & 2 & 107 & $0.91(0.80-1.00)$ & 0.69 & $0.82(0.53-1.00)$ & 0.09 \\
\hline Other races & 14 & 754 & $0.87(0.80-0.93)$ & & $0.97(0.95-1.00)$ & \\
\hline \multicolumn{7}{|l|}{ Type of study } \\
\hline Prospective & 5 & 301 & $0.93(0.88-0.98)$ & 0.61 & $0.99(0.96-1.00)$ & 0.08 \\
\hline Retrospective & 8 & 473 & $0.79(0.72-0.86)$ & & $0.93(0.87-1.00)$ & \\
\hline \multicolumn{7}{|l|}{ Etiology } \\
\hline AMD & 7 & 560 & $0.89(0.82-0.95)$ & 0.12 & $0.98(0.94-1.00)$ & 0.44 \\
\hline Other ocular diseases & 9 & 301 & $0.85(0.76-0.94)$ & & $0.97(0.92-1.00)$ & \\
\hline \multicolumn{7}{|l|}{ Reference standard } \\
\hline FA & 9 & 549 & $0.83(0.76-0.90)$ & $<0.01$ & $0.94(0.88-1.00)$ & 0.09 \\
\hline Other reference standards & 7 & 372 & $0.92(0.86-0.97)$ & & $0.99(0.97-1.00)$ & \\
\hline \multicolumn{7}{|l|}{ Type of device } \\
\hline AngioVue & 10 & 426 & $0.90(0.86-0.95)$ & 0.09 & $0.96(0.91-1.00)$ & 0.66 \\
\hline other devices & 6 & 435 & $0.80(0.72-0.87)$ & & $0.98(0.96-1.00)$ & \\
\hline \multicolumn{7}{|l|}{ Sample size } \\
\hline$\geq 50$ & 8 & 690 & $0.88(0.81-0.95)$ & 0.12 & $0.96(0.91-1.00)$ & 0.57 \\
\hline$<50$ & 8 & 171 & $0.86(0.77-0.96)$ & & $0.99(0.96-1.00)$ & \\
\hline
\end{tabular}

invasive dye technology, with risks of side effects after injecting intravenous contrast agents [36]. In spite of accurate identification of CNV patients, the invasive injury could not be avoided for suspected patients without $\mathrm{CNV}$. In addition, OCT is also a technology of detecting neovascular AMD, but it is not a single diagnostic tool owing to limitations in sensitivity and specificity [37]. Compared with OCT, OCTA is a more optimal technology and has been widely applied in the clinical recently. At present, AMD appears to be the most common disease, which might develop CNV. Therefore, in this study, seven articles were collected and analyzed, including 560 eyes that reported the diagnostic value of OCTA in only AMD related CNV. The sensitivity and specificity of OCTA to AMD related CNV were not significantly different from those of other ocular diseases $(p=0.12$ and $p=0.44)$. In AMD patients, the occurrence of $\mathrm{CNV}$ in one eye may lead to CNV risk in the other eye. In a randomized clinical trial, 727 patients with $\mathrm{CNV}$ in one single eye were enrolled to receive a ranibizumab or bevacizumab therapy for the other eye. Two years after the treatment, $19 \%$ patients developed CNV in the other eye [38]. Thus, if OCTA technology could be performed to monitor $\mathrm{CNV}$ in the other eye, $88 \%$ of post-test probability in CNV positive eyes and 3\% of post-test probability in $\mathrm{CNV}$ negative eyes would be reached. Together, these findings demonstrated that OCTA was a reliable imaging technology in early detection of $\mathrm{CNV}$ in AMD patients who had developed a CNV eye, which was also able to exclude individuals without $\mathrm{CNV}$. For suspicious $\mathrm{CNV}$ eyes, it is unrealistic to receive constant dye angiography for monitoring disease progression, rather, it is promising to use OCTA as a surveillance and screening method.

In general, types of both study and device were potential sources of overall heterogeneity in this meta-analysis. The diagnostic value between the five prospective studies including 305 eyes was significantly different from that in the eight prospective studies including 473 eyes $(p<0.01)$. Compared with prospective studies, retrospective studies might underestimate diagnostic value due to the possible biases caused by inaccurate information recall by researchers. Moreover, to some extent, different kinds of devices might lead to imaging differences in diagnosis. As we have known, the projection artifact is a common problem affecting the diagnostic accuracy of the image equipment in detecting diseases. One of the reasons for projection artifacts is associated with the imaging equipment itself. The difference between AngioVue device group including 10 studies (426 eyes) is significantly different from other devices group including six studies (435 eyes) ( $P$-value was 0.04$)$. The sensitivity and specificity of AngioVue group were 0.90 and 0.96 , respectively, while they were 0.83 and 0.98 , respectively in other devices group. In addition, the reference standard was a source of heterogeneity in sensitivity between only FA group and other reference standards group $(p<0.01)$. The sensitivity in seven other reference standards studies including 312 eyes was higher than nine FA studies including 
549 eyes (0.92 versus 0.83 ). Of note, FA is a common reference standard of the diagnosis of ocular vascular diseases due to the advantage of showing the leakage of neovascularization. The principle of OCTA is different from FA, for it is a quantitative equipment and provides neovascular network information. Souedan V et al used the multimodal imaging as the diagnostic reference standard to assess the diagnostic value of OCTA for CNV [30]. As a result, they found that OCTA showed a higher diagnostic value than FA, for the sensitivity and specificity of OCTA were 85.62 and $81.51 \%$, respectively, and the sensitivity and specificity of FA for CNV were 74.5 and $82.35 \%$, respectively. Besides, compared with FA, other clinical diagnostic methods such as ICGA and OCT also have their own advantages. Other reference standards including multimodal imaging may contribute to the correct classification of diseases, which may lead to a higher sensitivity in other reference standards group in our study.

Additionally, the difference between Asian people and other racial people was also analyzed in the present study. Differences in sensitivity, specificity and overall diagnostic value of ethnicity were not statistically significant, for $P$-values were $0.69,0.09$ and 0.10 , respectively. In consideration of the influence of sample size, 16 articles were divided into two subgroups based on whether the sample size of each study was less than 50 . As a result, there was no significant difference, thus, sample size was not the source of heterogeneity.

There were certain limitations in our meta-analysis based on published articles. To begin with, in this study, we included all types of $\mathrm{CNV}$ to analyze the overall diagnostic value for all kinds of CNV. The diagnostic value of OCTA for different types of CNV might be different, however, the information of each study was insufficient to analyze the difference. Thus, we failed to analyze whether the type of CNV was the source of heterogeneity. Secondly, the sample size of several studies was less than 50 and some studies were retrospective ones in our meta-analysis. To this end, more prospective studies with large sample are warranted to confirm the diagnostic ability of OCTA for CNV in the future. Thirdly, only two studies reported the diagnostic value of OCTA only in Asian population, therefore, more studies from different countries are expected in the future.

In conclusion, OCTA is a potential and reliable method for detecting $\mathrm{CNV}$ in ocular diseases based on the sensitivity, specificity and AUC. It might be conducive to monitor the other eye in the case of $\mathrm{CNV}$ presence in one eye. In addition, more longitudinal studies of large sample are urgently needed to confirm this conclusion.

\section{Abbreviations}

AMD: Age-related macular degeneration; CNV: Choroidal neovascularization; CSCR: Central serous chorioretinopathy; DOR: Diagnostic odds ratio; FA: Fluorescein angiography; ICGA: Indocyanine green angiography; NLR: Negative likelihood ratio; OCT: Optical coherence tomography; OCTA: Optical coherence tomography angiography; PLR: Positive likelihood ratio; PRISMA: Preferred Reporting Items for Systematic Reviews and Meta-analysis; QUADAS-2: Quality Assessment of diagnostic Accuracy Version 2; SROC: The summary receiver operating characteristic; SROC-AUC: The area under the sROC curve

\section{Acknowledgments}

Not applicable.

\section{Authors' contributions}

WR, LZZ and LX participated in the completion of this manuscript. WR, LZZ and LX contributed to screen literature, evaluated the quality of articles and extracted data. WR conducted statistical analysis and wrote the manuscript. LX revised the manuscript. All authors read and approve the final version.

\section{Funding}

This study was supported by the National Key Research and Development Program of China (Grant No. 2017YFC0108602) and the Technology Project of the Department of Health of Jilin Province (Grant No. 20165044). The collection, analysis, and interpretation of data were supported by the National Key Research and Development Program of China (Grant No. 2017YFC0108602) The design of this study and the writing of this manuscript were supported by the Technology Project of the Department of Health of Jilin Province (Grant No. 20165044)

\section{Availability of data and materials}

All data in this study was available, for they were all collected from published articles through electronic databases.

Ethics approval and consent to participate

Not applicable.

\section{Consent for publication}

Not applicable.

\section{Competing interests}

The authors declare that they have no competing interests.

\section{Author details}

${ }^{1}$ Epidemiology and Statistics, School of Public Health, Jilin University, Changchun 130021, China. ${ }^{2}$ Department of NHC Key Laboratory of Radiation Biology, Jilin University, Changchun 130021, China.

Received: 14 April 2019 Accepted: 9 July 2019

Published online: 26 July 2019

\section{References}

1. Kashani AH, Chen CL, Gahm JK, Zheng F, Richter GM, Rosenfeld PJ, et al. Optical coherence tomography angiography: a comprehensive review of current methods and clinical applications. Prog Retin Eye Res. 2017:60:66-100

2. Spaide RF, Fujimoto JG, Waheed NK, Sadda SR, Staurenghi G. Optical coherence tomography angiography. Prog Retin Eye Res. 2018:64:1-55.

3. Neelam K, Cheung CM, Ohno-Matsui K, Lai TY, Wong TY. Choroidal neovascularization in pathological myopia. Prog Retin Eye Res. 2012;31(5): 495-525.

4. Chan WM, Lam DS, Lai TY, Yuen KS, Liu DT, Chan CK, et al. Treatment of choroidal neovascularization in central serous chorioretinopathy by photodynamic therapy with verteporfin. Am J Ophthalmol. 2003;136(5):836-45

5. van Lookeren CM, LeCouter J, Yaspan BL, Ye W. Mechanisms of agerelated macular degeneration and therapeutic opportunities. J Pathol. 2014:232(2):151-64.

6. Baxter SL, Pistilli M, Pujari SS, Liesegang TL, Suhler EB, Thorne JE, et al. Risk of choroidal neovascularization among the uveitides. Am J Ophthalmol. 2013;156(3):468-77.e2 
7. Campochiaro PA. Molecular pathogenesis of retinal and choroidal vascular diseases. Prog Retin Eye Res. 2015:49:67-81.

8. Grossniklaus HE, Green WR. Choroidal neovascularization. Am J Ophthalmol. 2004;137(3):496-503.

9. Wong CW, Yanagi Y, Lee WK, Ogura Y, Yeo I, Wong TY, et al. Age-related macular degeneration and polypoidal choroidal vasculopathy in Asians. Prog Retin Eye Res. 2016;53:107-39.

10. Holden BA, Fricke TR, Wilson DA, Jong M, Naidoo KS, Sankaridurg P, et al. Global prevalence of myopia and high myopia and temporal trends from 2000 through 2050. Ophthalmology. 2016;123(5):1036-42.

11. Colijn JM, Buitendijk GHS, Prokofyeva E, Alves D, Cachulo ML, Khawaja AP, et al. Prevalence of age-related macular degeneration in Europe: the past and the future. Ophthalmology. 2017;124(12):1753-63.

12. Yoshida T, Ohno-Matsui K, Yasuzumi K, Kojima A, Shimada N, Futagami S, et al. Myopic choroidal neovascularization: a 10-year follow-up. Ophthalmology. 2003;110(7):1297-305.

13. Amissah-Arthur KN, Panneerselvam S, Narendran N, Yang YC. Optical coherence tomography changes before the development of choroidal neovascularization in second eyes of patients with bilateral wet macular degeneration. Eye (Lond). 2012;26(3):394-9.

14. Ferrara D, Waheed NK, Duker JS. Investigating the choriocapillaris and choroidal vasculature with new optical coherence tomography technologies. Prog Retin Eye Res. 2016;52:130-55.

15. Leeflang MM, Deeks JJ, Gatsonis C, Bossuyt PM. Systematic reviews of diagnostic test accuracy. Ann Intern Med. 2008;149(12):889-97.

16. Whiting PF, Rutjes AW, Westwood ME, Mallett S, Deeks JJ, Reitsma JB, et al. QUADAS-2: a revised tool for the quality assessment of diagnostic accuracy studies. Ann Intern Med. 2011;155(8):529-36.

17. Moult E, Choi W, Waheed NK, Adhi M, Lee B, Lu CD, et al. Ultrahigh-speed swept-source OCT angiography in exudative AMD. Ophthalmic Surg Lasers Imaging Retina. 2014;45(6):496-505.

18. Lupidi M, Coscas G, Cagini C, Coscas F. Optical coherence tomography angiography of a choroidal neovascularization in adult onset Foveomacular Vitelliform dystrophy: pearls and pitfalls. Invest Ophthalmol Vis Sci. 2015; 56(13):7638-45.

19. Bonini Filho MA, de Carlo TE, Ferrara D, Adhi M, Baumal CR, Witkin AJ, et al. Association of Choroidal Neovascularization and Central Serous Chorioretinopathy with Optical Coherence Tomography Angiography. JAMA Ophthalmol. 2015;133(8):899-906.

20. de Carlo TE, Bonini Filho MA, Chin AT, Adhi M, Ferrara D, Baumal CR, et al. Spectral-domain optical coherence tomography angiography of choroidal neovascularization. Ophthalmology. 2015;122(6):1228-38.

21. Shaimov TB, Panova IE, Shaimov RB, Shaimovsmalla CVA, Shai Mova TA, Fomin AV, et al. Optical coherence tomography angiography in the diagnosis of neovascular age-related macular degeneration. Vestn oftalmol. 2015;131(5):4-13.

22. Carnevali A, Cicinelli MV, Capuano V, Corvi F, Mazzaferro A, Querques L, et al. Optical coherence tomography angiography: a useful tool for diagnosis of treatment-naive quiescent choroidal neovascularization. Am J Ophthalmol. 2016;169:189-98.

23. Miyata M, Ooto S, Hata M, Yamashiro K, Tamura H, Akagi-Kurashige Y, et a. Detection of myopic choroidal neovascularization using optical coherence tomography angiography. Am J Ophthalmol. 2016;165:108-14.

24. de Carlo TE, Rosenblatt A, Goldstein M, Baumal CR, Loewenstein A, Duker JS. Vascularization of irregular retinal pigment epithelial detachments in chronic central serous chorioretinopathy evaluated with OCT angiography. Ophthalmic Surg Lasers Imaging Retina. 2016;47(2):128-33.

25. Gong J, Yu S, Gong Y, Wang F, Sun X. The diagnostic accuracy of optical coherence tomography angiography for Neovascular age-related macular degeneration: a comparison with fundus fluorescein angiography. J Ophthalmol. 2016;2016:7521478.

26. Querques L, Giuffre C, Corvi F, Zucchiatti I, Carnevali A, De Vitis LA, et al. Optical coherence tomography angiography of myopic choroidal neovascularisation. Br J Ophthalmol. 2017:101(5):609-15.

27. Faridi A, Jia Y, Gao SS, Huang D, Bhavsar KV, Wilson DJ, et al. Sensitivity and specificity of OCT angiography to detect choroidal neovascularization. Ophthalmology retina. 2017;1(4):294-303.

28. Ahmed D, Stattin M, Graf A, Forster J, Glittenberg C, Krebs I, et al. Detection of treatment-naive choroidal neovascularization in age-related macular degeneration by swept source optical coherence tomography angiography. Retina (Philadelphia, Pa). 2018;38(11):2143-9
29. Nikolopoulou E, Lorusso M, Ferrari LM, Cicinelli MV, Bandello F, Querques G, et al. Optical coherence tomography angiography versus dye angiography in age-related macular degeneration: sensitivity and specificity analysis. Biomed Res Int. 2018;2018(Article ID 6724818):1-7.

30. Souedan V, Souied EH, Caillaux V, Miere A, El Ameen A, Blanco-Garavito R. Sensitivity and specificity of optical coherence tomography angiography (OCT-A) for detection of choroidal neovascularization in real-life practice and varying retinal expertise level. Int Ophthalmol. 2018;38(3):1051-60.

31. Soomro T, Talks J. The use of optical coherence tomography angiography for detecting choroidal neovascularization, compared to standard multimodal imaging. Eye (Lond). 2018;32(4):661-72.

32. de Oliveira T, Isaac DLC, JMBdB G, Schelini MC, Avila MP. Oct angiography compared to fluorescein angiography, indocyanine green angiography and optical coherence tomography in the detection of choroidal neovascularization in pigment epithelial detachment. Acta Ophthalmol. 2019. https://doi.org/10.1111/aos.14117.

33. Wong WL, Su X, Li X, Cheung CM, Klein R, Cheng CY, et al. Global prevalence of age-related macular degeneration and disease burden projection for 2020 and 2040: a systematic review and meta-analysis. Lancet Glob Health. 2014;2(2):e106-16.

34. Villegas VM, Aranguren LA, Kovach JL, Schwartz SG, Flynn HW Jr. Current advances in the treatment of neovascular age-related macular degeneration. Expert Opin Drug Deliv. 2017;14(2):273-82

35. Stem MS, Moinuddin O, Kline N, Thanos A, Rao P, Williams GA, et al. Outcomes of anti-vascular endothelial growth factor treatment for choroida neovascularization in fellow eyes of previously treated patients with Neovascular age-related macular degeneration. JAMA Ophthalmol. 2018; 136(7):820-3.

36. Su Z, Ye $P$, Teng $Y$, Zhang $L$, Shu $X$. Adverse reaction in patients with drug allergy history after simultaneous intravenous fundus fluorescein angiography and indocyanine green angiography. J Ocul Pharmacol Ther. 2012;28(4):410-3.

37. Mowatt G, Hernandez R, Castillo M, Lois N, Elders A, Fraser C, et al. Optical coherence tomography for the diagnosis, monitoring and guiding of treatment for neovascular age-related macular degeneration: a systematic review and economic evaluation. Health Technol Assess (Winch Eng). 2014; 18(69):1-254.

38. Maguire MG, Daniel E, Shah AR, Grunwald JE, Hagstrom SA, Avery RL, et al. Incidence of choroidal neovascularization in the fellow eye in the comparison of age-related macular degeneration treatments trials. Ophthalmology. 2013;120(10):2035-41.

\section{Publisher's Note}

Springer Nature remains neutral with regard to jurisdictional claims in published maps and institutional affiliations.

Ready to submit your research? Choose BMC and benefit from:

- fast, convenient online submission

- thorough peer review by experienced researchers in your field

- rapid publication on acceptance

- support for research data, including large and complex data types

- gold Open Access which fosters wider collaboration and increased citations

- maximum visibility for your research: over $100 \mathrm{M}$ website views per year

At BMC, research is always in progress.

Learn more biomedcentral.com/submissions 